\title{
Mediastinal foregut duplication cyst of enteric type containing a persistent thymus, imitating a pericardial cyst
}

\author{
Dubrava $\mathrm{J}^{1}$, Koren $\mathbf{J}^{2}$, Pospisilova $\mathrm{V}^{3}$ \\ Department of Noninvasive Cardiology, St. Cyril and Method Hospital, University Hospital Bratislava, Slovakia. \\ dubrava@pe.unb.sk
}

\begin{abstract}
Mediastinal foregut duplication cysts are rare congenital masses caused by developmental disorders of the anterior part of the embryonic primitive gut. In adults they can be discovered as an incidental finding on chest radiograph. They can mimic other intrathoracic pathologies as it was initially the case in our patient. A 51-year-old woman was incidentally found to have homogenous mass at the right cardiophrenic angle on the chest radiograph. Based on that finding and echocardiography a pericardial cyst was suspected. Computed tomography demonstrated a simple anterior mediastinal cyst. Because of the growth with a mild progression of the compression of the right atrium, the cyst was resected. Histology and immunohistochemistry revealed the mass to be a mediastinal foregut duplicatory cyst of enteric type containing persistent thymus. To our knowledge, this is the first report in which a mediastinal foregut cyst contained both enteric mucosa and ectopic persistent thymus (Fig. 9, Ref. 13). Full Text in PDF www.elis.sk.

Key words: foregut cyst, mediastinal cyst, thymus, enteric cyst, pericardial cyst.
\end{abstract}

The foregut is the anterior part of the embryonic primitive gut. Mediastinal foregut duplication cysts are uncommon congenital masses caused by developmental disorders of the foregut. They comprise approximately $10 \%$ of adult mediastinal tumors and fewer than $5 \%$ of mediastinal masses in children (1). The wall of these rare congenital anomalies is composed of a fibrous connective tissue with a well developed smooth muscle layer and the inner surface of the cyst is lined by epithelium. In adults they are frequently discovered as an incidental finding on chest radiograph. Mediastinal foregut cysts can mimic other intrathoracic pathologies as was initially suspected in our patient.

\section{Case report}

A 51-year-old woman was incidentally found to have homogenous mass at the right cardiophrenic angle on chest radiograph (Fig. 1). She had no complaints. Physical examination was unremarkable. The ECG was normal. Transthoracic and transesophageal echocardiography showed an oval-shaped cystic mass measuring $85 \times 82 \times 56 \mathrm{~mm}$, closely attached to the right atrium, right ventricle, and superior vena cava (Fig. 2). The echocardiogram demonstrated a mild compression of the right atrium. Intravenous echocontrast excluded a communication between the mass and the

\footnotetext{
${ }^{1}$ Department of Noninvasive Cardiology, St. Cyril and Method Hospital, University Hospital Bratislava, Slovakia, ${ }^{2}$ Department of Pathology, Slovak Medical University, Bratislava, Slovakia, and ${ }^{3}$ Institute of Histology and Embryology, Faculty of Medicine, Comenius University, Bratislava, Slovakia

Address for correspondence: J. Dubrava, MD, PhD, Department of Noninvasive Cardiology, St. Cyril and Method Hospital, Antolska 11, SK-851 07 Bratislava, Slovakia.

Phone: +421.2.68672319, Fax: +421.2 .68672029$
}

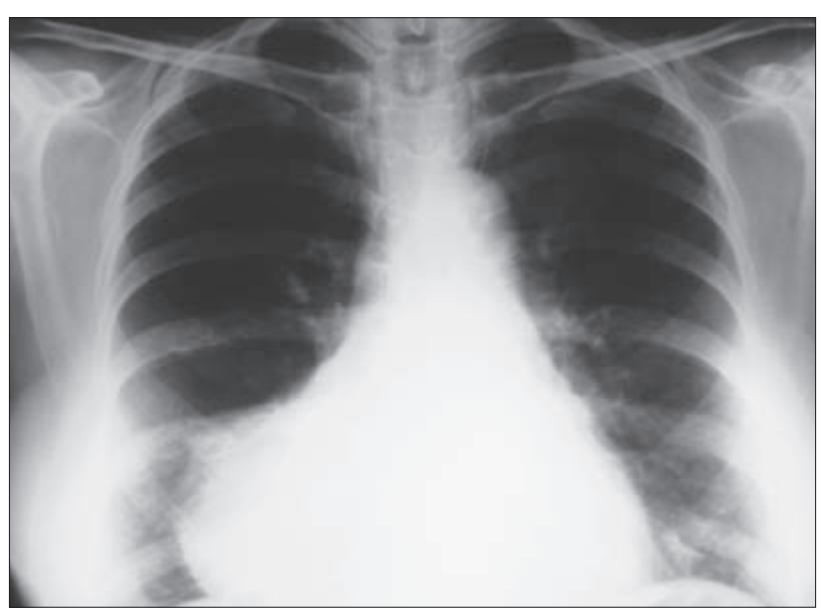

Fig. 1. The posteroanterior chest radiograph demonstrates a homogenous mass at the right cardiophrenic angle.

right heart chambers. A small foramen ovale with a mild right-toleft shunt was found. Based on echocardiography and chest radiograph pericardial cyst was suspected. Computed tomography demonstrated a simple anterior mediastinal cyst with the size of $90 \times 72 \times 60 \mathrm{~mm}$ (Fig. 3). Because of patient's profession (veterinary surgeon) echinococcosis was suspected, but excluded with negative serology. During a 3-year follow-up a mild growth of the cyst with a mild progression of right atrial compression was observed. The patient prefered mass removal and she underwent resection of the cyst. It was found originating from the middle mediastinum and spreading cranially to the thymic area. The cyst was attached to the outside surface of pericardium. Positivity of pan cytokeratin antibody [AE1/AE3] with cyst lining confirmed that its internal layer constituted an epithelium (Fig. 4). Cyst wall was lined by 


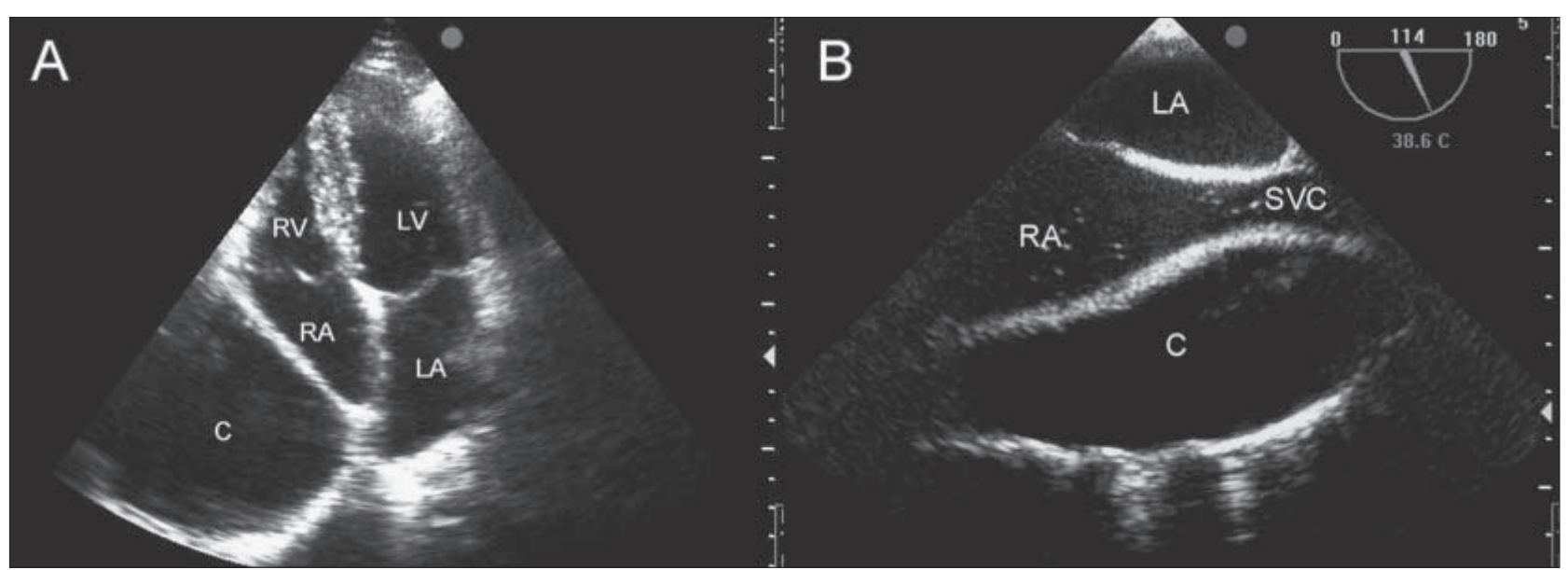

Fig. 2. Transthoracic (A) and transesophageal (B) echocardiography showing an oval-shaped cystic mass (C), closely attached to the right atrium (RA), right ventricle (RV) and superior vena cava (SVC). The rests of intravenous echocontrast in the right heart in the (B) view. LA - left atrium, $L V$ - left ventricle.

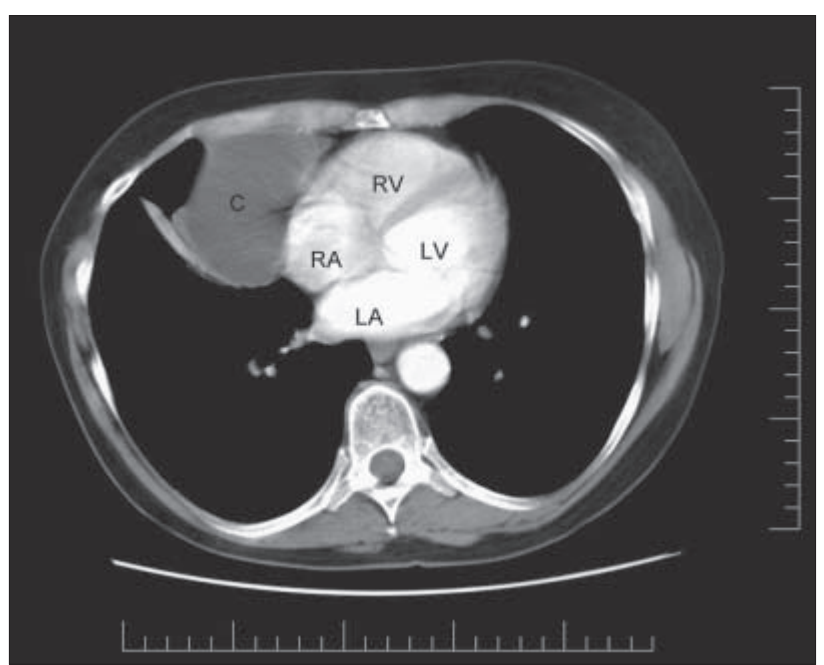

Fig. 3. CT contrast examination demonstrates a simple anterior mediastinal cyst (C). LA - left atrium, LV - left ventricle, RA - right atrium, RV - right ventricle.

simple columnar epithelium of enteric type (Fig. 5). Immunohistochemical negativity with calretinin antibody excluded mediastinal cystic mesothelioma. Histology and immunohistochemistry revealed the mass to be a mediastinal foregut duplicatory cyst of enteric type containing persistent thymus. A thymus tissue with multiple Hassall's corpuscles was present at several locations of the cyst wall (Fig. 6). Hassall's corpuscles were positive for pan cytokeratin antibody [AE1/AE3] (Fig. 7). The study methods revealed thymic cortical and medullar structures and also cortical stromal epithelio-reticular cells positive for cytokeratin antibody [AE1/AE3]. Diffuse immunopositivity for CD45RO in T lymphocytes was found within the thymic tissue (Fig. 8). Immunopositivity for CD20 in B lymphocytes of a thymic medullary structures is shown in Figure 9.

The patient has remained asymptomatic and free of relapse of the mediastinal mass three years after the surgery.

\section{Discussion}

Early development of the thymus is closely connected with the development of a primitive gut of a human embryo. The majority of the primitive gut develops from the embryonic endoderm of the germ disc. During the embryonic life, the primitive gut can be divided into three segments: foregut, midgut and hindgut. By convention, the boundaries of the foregut, midgut and hindgut correspond to the territories of the three arteries that supply abdominal gut tube (celiac trunk, superior mesenteric trunk and inferior mesenteric artery). The most cranial part of the foregut - so called pharyngeal gut forms primordia of the pharynx and pharyngeal organs. The next part of the foregut orally starts from the respiratory diverticulum (primordium of respiratory tract including the lungs) to the entrance of the bile duct. This anterior part of the alimentary canal gives rise to the esophagus, stomach, upper duodenum (1st and 2nd parts), liver, gallbladder and pancreas.

Five pairs of pharyngeal arches form on either side of the pharyngeal gut (initially they resemble the gills of a fish). The arches are separated externally by ectoderm-lined clefts and internally by endoderm-lined pharyngeal pouches. Each pharyngeal pouche gives rise to an adult structure - 1st: tympanic cavity and Eustachian tube, 2nd: palatine tonsils, 3rd: thymus gland and parathyroid glands. The right and left thymic primordia descend as a duct (ductus thymicus) and come into apposition forming the one organ near to the pericardial cavity (2). The pharyngeal clefts include epibranchial neuroplacodes which also play a role in the thymus organogenesis (3). The organogenesis of human thymus depends on the interactions between endodermal epithelium of pharyngeal pouches, ectodermal epithelium and neuroplacodes of pharyngeal clefts, neural crest-derived mesenchyme (3) and mesoderm-derived hematopoietic cells and vessels (4).

Because the thymus development is complex and complicated several developmental disorders of this organ exist. A remnant of the thymic tissue may persist in the neck along the pathway of descent (thymus cervicalis). A portion of the thymus may become 


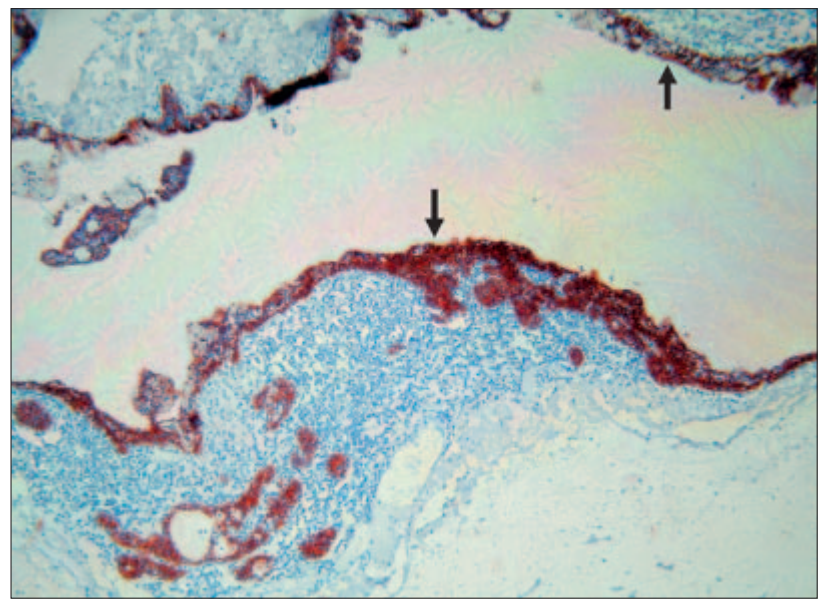

Fig. 4. Positivity of pan cytokeratin antibody (AE1/AE3) with cyst lining (arrows) revealed that the internal layer constituted an epithelium (immunohistochemistry, original magnification x 100).

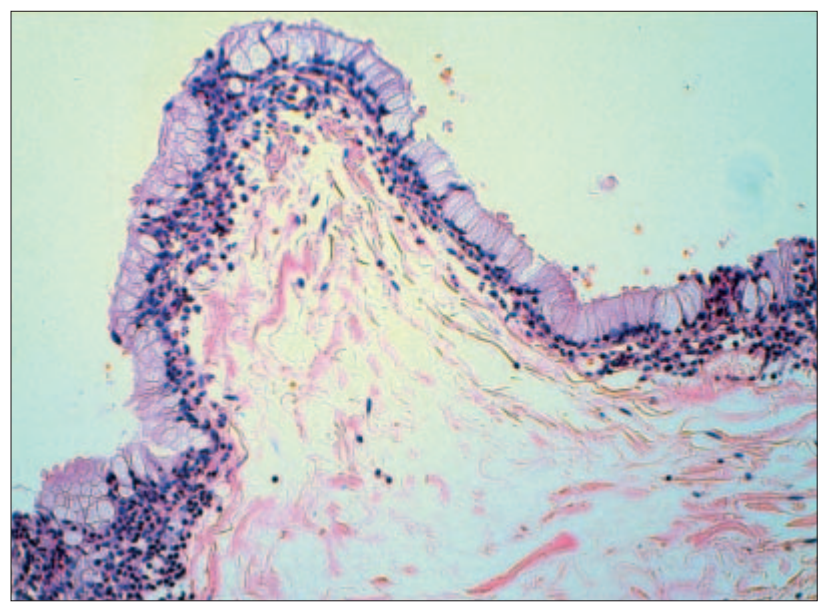

Fig. 5. Cyst wall at higher magnification. It is shown to be lined by mucous single-layer columnar epithelium of enteric type (hematoxylin and eosin, original magnification $x$ 500).

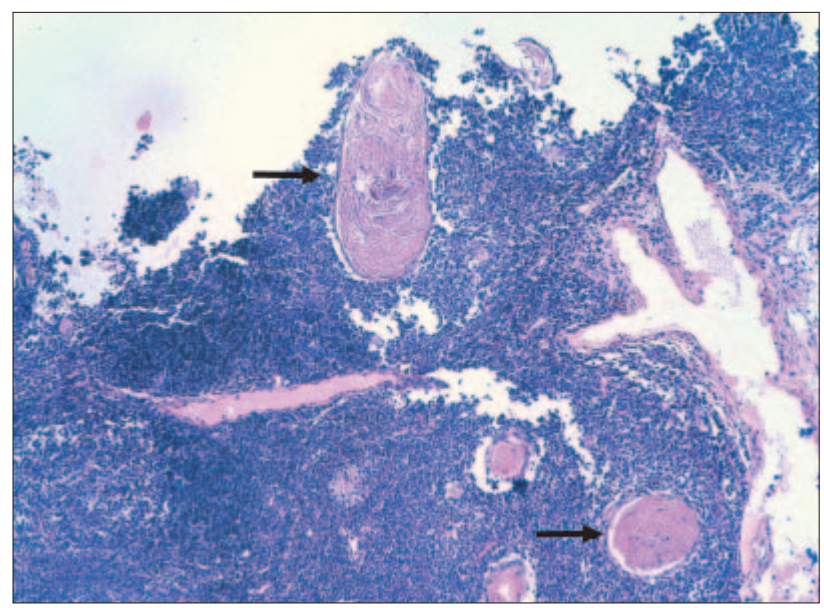

Fig. 6. Histology revealing a persistent thymus with multiple large Hassall's corpuscles (arrows) at a cyst wall (hematoxylin and eosin, original magnification $\mathrm{x} 250$ ).

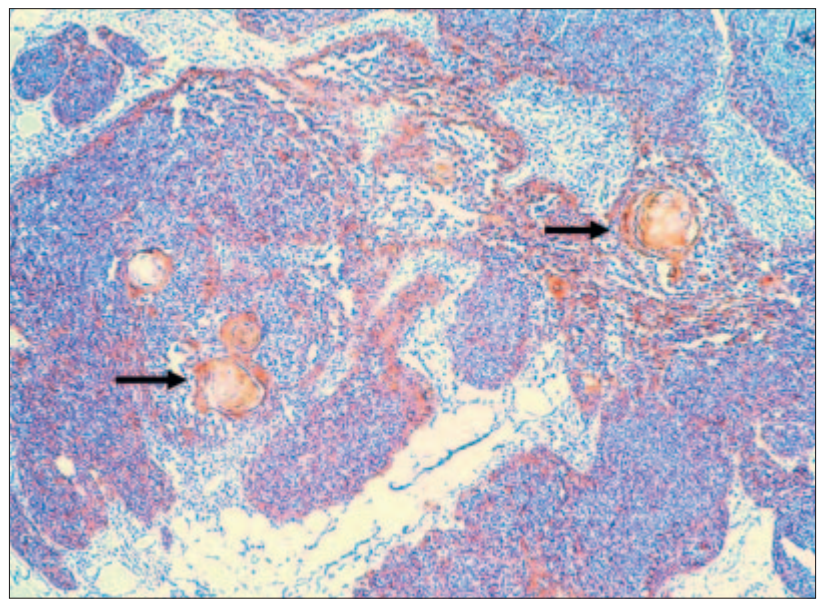

Fig. 7. Positivity of pan cytokeratin antibody (AE1/AE3) with Hassall's corpuscles (arrows) and cortical reticulo-epithelial cells (immunohistochemistry, original magnification $x$ 250).

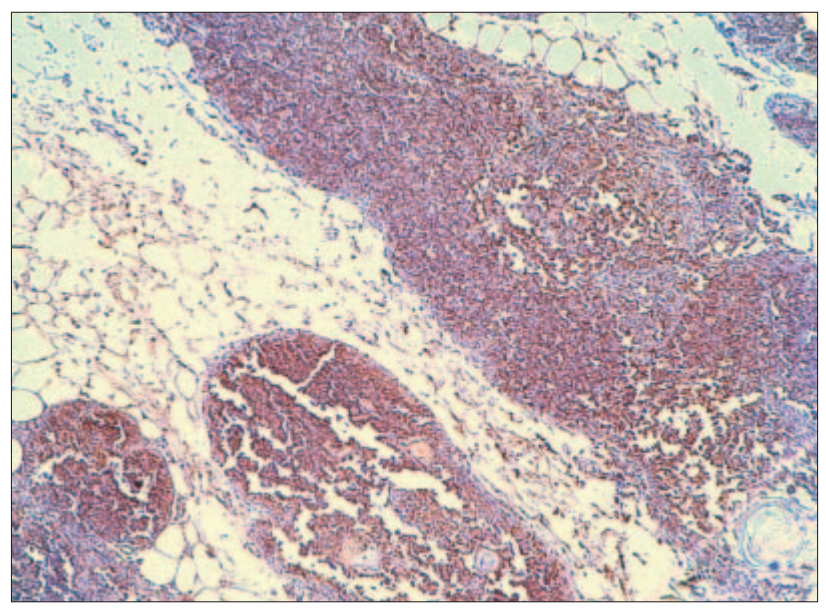

Fig. 8. Diffuse immunopositivity for CD45RO in $T$ lymphocytes in a thymus (immunohistochemistry, original maginification x 250).

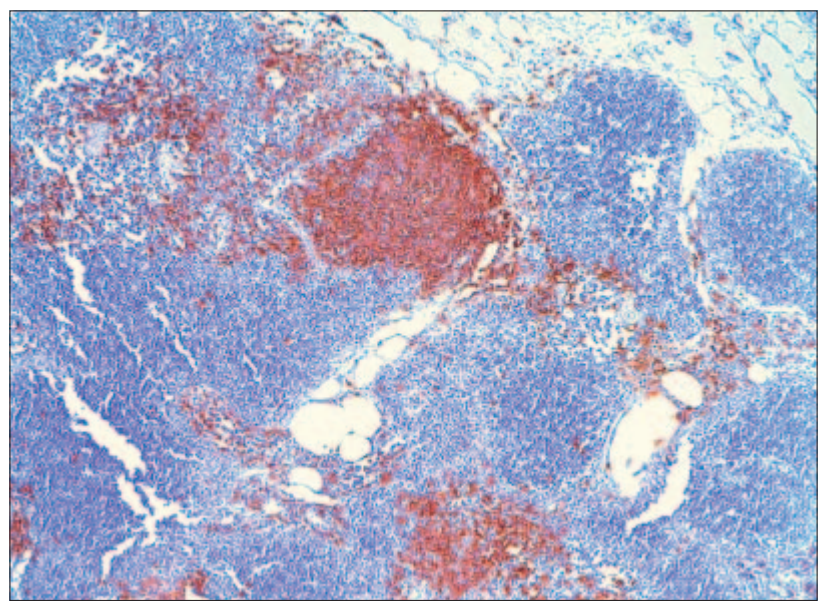

Fig. 9. Immunopositivity for CD20 in B lymphocytes of a thymic medulla (immunohistochemistry, original magnification x 250). 
separated from the main organ during the development (accessory thymus). The disruption of thymic descent from pharyngeal region to the anterior mediastinum can result in an ectopic thymus localized anywhere from the mandibular angle to the upper mediastinum. About 100 cases have been described in the literature, $10 \%$ of which occurred in neonates. Ectopic thymus does not usually cause significant symptoms. It can mimick thyroid gland enlargement or subglottic mass as a congenital haemangioma (5). Even rarer is the thymopharyngeal duct cyst. It may develop from the wall of pharynx and thymus primordium (6). They have been described as asymptomatic and of little clinical consequence. Respiratory compromise associated with these lesions is possible. Thymopharyngeal duct cysts are usually present in the 1st decade of life, after the age of two years, possibly because thymus attains its greatest development before puberty. Since it is rare to diagnose this entity before its surgical removal, the differential diagnosis includes the more common pharyngeal cleft cyst, thyroglossal duct cyst, cystic hygroma, cystic dermoid and lesions of the salivary gland, thyroid and parathyroid glands and cervical lymph nodes (7). The developmental anomalies, mentioned above, may be found anywhere along the normal descent route of the thymus gland from the mandible to the manubrium sterni; $50 \%$ of them extend into the mediastinum $(8,9)$.

To our knowledge we presented the first case of a persistent ectopic thymus incorporated in a mediastinal foregut cyst containing enteric mucosa. Congenital duplicatory foregut cysts are caused by the developmental disorders of the foregut. Mediastinal foregut cysts include bronchogenic, esophageal, neurenteric cysts (1). Before the obliteration of the dorsal mesocardium between the 7 th and 16th somatic stages, the foregut is in close proximity to the developing heart (10). Mediastinal foregut cysts can be therefore localized in the close proximity of the heart as it was in our patient.

Presenting symptoms of mediastinal cysts are variable and they may lead to diagnostic confusion. Intrathoracic foregut duplication cysts present most commonly with oesophageal or airway obstruction, haematemesis, melaena or haemoptysis. Diagnostic delay is common (11). In adults, mediastinal foregut cysts are frequently asymptomatic and they can be initially discovered as an incidental finding on chest radiography. The most common appearance is that of a round or oval soft tissue mass. Additional radiographic signs are secondary to extrinsic compression of vascular and bronchial structures by the cyst (1). The differentiation of the mediastinal cysts from other mediastinal masses is important and not allways simple. The final diagnosis is frequently made only after histopathological examination. We illustrate this by two cases of mediastinal masses associated with thymus pathology. Caramori et al. reported a case of thymoma at the right cardiophrenic angle. Similarly as in our case the mass was initially suspected at echocardiography to be a pericardial cyst. The diagnosis was made only histopathologically (12). Tanimura et al described a case of a large anterior mediastinal cyst with a small mass on a part of its wall. The pathological diagnosis was a thymic basaloid carcinoma originating from the wall of a thymic cyst (13).

The therapy of mediastinal cysts depends mainly on the symptomatology. Surgical resection of mediastinal foregut cysts is necessary in symptomatic patients. For asymptomatic patients with a round to oval, nonenhancing, thin walled, cystic mass demonstrated on CT some authors recommend conservative radiographic follow-up (1).

\section{References}

1. Fitch SJ, Tonkin IL, Tonkin AK. Imaging of foregut duplication cysts. Radiographics 1986; 6 (2): 189-201.

2. Slípka J, Pospíšilová V, Slípka J Jr. Evolution, development and involution ot the thymus. Folia Microbiol (Praha) 1998; 43 (5): 527-530.

3. Pospíšilová V, Slípka J, Černý R. The relation of the germ layers to the ecto-mesenchyme of the neural placodes during thymus development. Plzeň lék Sborn 2003; Suppl. 78: 23-28.

4. Varga I, Pospisilova V, Jablonska-Mestanova V, Galfiova P, Polak S. The thymus: Picture review of human thymus prenatal development. Bratisl Lek Listy 2011; 112 (7): 368-376.

5. Varga I, Uhrinova A, Toth F, Mistinova J. Assessment of the thymic morphometry using ultrasound in full-term newborns. Surg Radiol Anat 2011; 33 (8): 689-695.

6. Varga I, Galfiova P, Jablonska-Mestanova V, Polak S, Adamkov M. Some aspects of early development of the thymus: embryological basis for ectopic thymus and thymopharyngeal duct cyst. Rev Arg Anat Clin 2011; 3 (1): 22-31.

7. Zarbo RJ, McClatchey KD, Areen RG, Baker SB. Thymopharyngeal duct cyst: a form of cervical thymus. Ann Otol Rhinol Laryngol 1983; 92 (3 Pt 1): 284-289.

8. De Caluwé D, Ahmed M, Puri P. Cervical thymic cysts. Pediatr Surg Int 2002; 18 (5-6): 477-479.

9. Cigliano B, Baltogiannis N, De Marco M et al. Cervical thymic cysts. Pediatr Surg Int 2007; 23 (12): 1219-1225.

10. Todd JL, Silverman ME, Kirby ML et al. Heart. 912-957. In: Skandalakis JE, Gray SW (Eds). Embryology for surgeons, 2nd edn. Baltimore; Williams and Wilkins, 1994.

11. Stringer MD, Dinwiddie R, Hall CM, Spitz L. Foregut duplication cysts: a diagnostic challenge. J R Soc Med 1993; 86 (3): 174-175.

12. Caramori G, Calia N, Pasquini $\mathbf{C}$ et al. Ectopic thymoma simulating a pericardial cyst. Monaldi Arch Chest Dis 2005; 63 (4): 230-233.

13. Tanimura S, Tomoyasu H, Kohno T, Matsushita H. Basaloid carcinoma originated from the wall of thymic cyst presenting as pericardial and thoracic effusion; report of a case. Kyobu Geka 2002; 55 (7): 571-575.

Received February 10, 2011. Accepted January 23, 2013. 